\title{
"3S" TECHNOLOGIES AND APPLICATION FOR DYNAMIC MONITORING SOIL AND WATER LOSS IN THE YANGTZE RIVER BASIN, CHINA
}

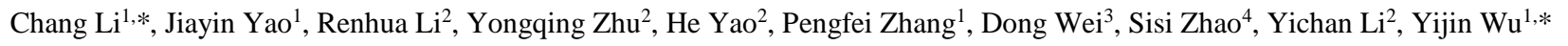 \\ ${ }^{1}$ Key Laboratory for Geographical Process Analysis \& Simulation, Hubei Province, and College of Urban and Environmental \\ Science, Central China Normal University, Wuhan, China, *lcshaka@126.com \& lichang@ mail.ccnu.edu.cn; *Corresponding author: \\ *wuyijin@mail.ccnu.edu.cn \\ ${ }^{2}$ Changjiang Soil and Water Conservation Monitoring Center CWRC, Wuhan, China \\ ${ }^{3}$ School of Remote Sensing and Information Engineering, Wuhan University, Wuhan 430079, China \\ ${ }^{4}$ Vivo Mobile Communication Co., Ltd
}

Commission III, ICWG III/7

\begin{abstract}
KEY WORDS: Soil and Water Loss, 3S (RS, GIS and GPS), Unmanned Aerial Vehicle (UAV), Dynamic Monitoring, Yangtze River Basin
\end{abstract}

\begin{abstract}
:
For China, which has many big rivers, there is an urgent need for efficient dynamic monitoring technology of water and soil loss. However, there are some problems in the current 3S (RS, GIS and GPS) technology for dynamic monitoring water and soil loss. This paper takes the Yangtze River Basin as an example to innovate and optimize the key technologies of the remote sensing interpretation of the water and soil loss dynamic monitoring of the Yangtze River Basin, and overcome the major technical difficulties in the remote sensing interpretation of the dynamic monitoring of water and soil loss. The key technologies include: 1) The establishment of a field investigation platform based on Internet and UAV (Unmanned Aerial Vehicle) for remote sensing interpretation; 2) Near real-time evaluating key factors of soil and water loss based on UAV photogrammetry and digital terrain analysis; 3) Geometric and Radiometric Simultaneous Correction Model (GRSCM) framework for remote sensing images preprocessing; 4) An object-oriented land use change update quality control method supported by multi-PC and GIS; and an objectoriented remote sensing image classification system based on random forest, deep learning and transfer learning; 5) Improvement of quantitative change detection method for image vegetation and three-dimensional topography. The results have been successfully applied in the remote sensing interpretation of the dynamic monitoring of water and soil loss in the national key prevention and control area of the Yangtze River Basin. They have been provided a scientific reference for the development planning of The Yangtze River Economic Zone.
\end{abstract}

\section{INTRODUCTION}

Water and soil loss is widespread in the world. Water erosion is by far the most important type of soil degradation affecting about 11 square kilometres $\left(\mathrm{km}^{2}\right)$ worldwide $(56 \%$ of the total area affected by human-induced soil degradation). The most widespread form of water erosion is the loss of the topsoil (on9.2 $\mathrm{km}^{2}$, while terrain deformation (rills and gullies) occurs on $1.75 \mathrm{~km}^{2}$ ( Oldeman, L. R. 1992). Water and soil loss is also one of the most serious ecological and environmental problems in China. It is caused by many factors such as climate, landform, geological structure, soil type, vegetation and human development. And it has caused great damage to the natural and social environment. In China, water and soil loss has destroyed land resources, exacerbated water pollution and induced ecological disasters. Today, China is experiencing rapid urbanization and modernization, and is facing challenges of population, resources and environmental issues. Water and soil loss has become one of the constraints of China's sustainable development. Therefore, it is necessary to monitor water and soil loss and soil and water conservation. The Yangtze River is the third largest river in the world and the largest river in China. It originates from the southwest side of the main peak of the Tanggula Mountains in the Qinghai-Tibet Plateau. The main stream has a total length of more than 6300 kilometres $(\mathrm{km})$, with the drainage basin of about 1.8 million $\mathrm{km}^{2}$ accounting for $18.8 \%$ of China's land area. The Yangtze River, with its huge river and lake systems and unique and complete natural ecosystems, is in an important position in China's economic and social development. Chinese President Xi Jinping emphasized the need to promote coordinated development of the upper, middle and lower reaches of the Yangtze River and high-quality development along the Yangtze River, making it an innovative demonstration zone for the implementation of protection and restoration of the ecological environment system. It shows that the dynamic monitoring of the water and soil loss of the Yangtze River and the corresponding prevention measures are an important links of water and soil loss control of in China. In order to reflect the water and soil loss status of the Yangtze River in a timely and comprehensive manner, the Changjiang Water Resources Commission of the Ministry of Water Resources of the People's Republic of China carried out dynamic monitoring of water and soil loss in 7 key national soil erosion prevention basins and $6 \mathrm{key}$ national soil erosion control basins in the Yangtze River Basin. The tasks of dynamic monitoring of water and soil loss in national key prevention and control basins of the drainage basin were fully covered.

When face the complicated geological environment, the traditional exploration methods in the field have too many difficulties to cope with, and it is difficult to enhance and ensure the overall level and quality of engineering geological investigation (Mao, Rui et al. 2011). It is a more efficient and convenient method to use remote sensing technology for dynamic monitoring of water and soil loss. However, at present, 
more than $95 \%$ of remote sensing images use human-computer interactive interpretation methods. There are many technical difficulties especially in field investigation and indoor interpretation.

The difficulties in the field investigation phase are: 1) Complicated operation. It is very inconvenient to carry a notebook, mouse, compass and hold GPS fixed point when setting up the field interpretation sign and recheck. 2) Vision blocked. Technicians cannot work in the mountainous terrain which has many ups and downs, limited horizons, and the blocked areas. 3) Different perspectives. The photos collected by the handheld device are at a horizontal viewing angle. While the satellite images on both sides of the ground objects are vertical in the process of vehicle driving, which leads to the difference of perspective in indoor interpretation. 4) Lack of near real-time evaluation. It is impossible to accurately obtain the three-dimensional information such as the slope and aspect of the sample at the same time in the field for the evaluation of the intensity of water and soil loss in this area. With the widespread application of " $3 \mathrm{~S}$ " technology, many experts use UAVs to collect data during field investigations. Although the UAV is convenient for the collection of remote sensing images and the establishment of field interpretation signs, its flight is greatly affected by weather, magnetic field, flight path and other objective conditions. These factors result in geometric distortion of the image, and even unqualified images such as stripes and distortions. At the later stage, it still needs manual pre-treatment before it can be used. While satellite imagery can provide high spatial resolution, it is temporally sparse and significant deformation can occur between observations (Parno, West et al. 2019). Therefore, the UAV remote sensing technology alone cannot fully realize the real-time data, nor can it be well combined with water and soil loss monitoring.

The bottlenecks in the indoor interpretation stage are: 1) $95 \%$ of the remote sensing images need to be interpreted manually. Not only is the labor intensity, speed, and labor cost high, but also the quality of the interpretation results is different, subject to strong personal subjective influence. 2) The traditional automatic classification system is not perfect, the automation is low, the features of the ground are not high, and the classification is inaccurate, resulting in low accuracy of the interpretation results, and even requiring manual second interpretation at a later stage, which seriously affects work efficiency. In recent years, various methods of Convolutional Neural Networks (CNN), Deep Learning, and Random forests have been used in feature recognition and classification. While some progress has been made, research in deep-learning-based remote-sensing image interpretation is still in its infancy, mainly subject to insufficient annotation samples, high complexity of the model, and lack of in-depth integration between deep learning and remote sensing ( $\mathrm{Li}$ et al. 2019).

Therefore, this paper proposes a system that integrates multiple classification algorithms and combines with the evaluation of water and soil loss factors, hoping to further improve the accuracy of feature recognition and simplify the process of water and soil loss evaluation.

Based on the technical difficulties of the above-mentioned dynamic detection of the water and soil loss of the Yangtze River for many years, this article proposes a series of innovative theories, methods and technologies, and develops corresponding prototype systems, hoping to improve the accuracy and speed of interpretation, and to improve remote sensing interpretation the scientific and timeliness of the results.

\section{METHODS AND TECHNILOGICAL PROCES}

\subsection{Field investigation}

2.1.1 A field investigation platform based on Internet and UAV for remote sensing interpretation: UAV - based surveys, including the image collection, processing, and visual interpretation, were considerably faster and more cost- efficient than ground - based surveys (Waite, van der Heijden et al. 2019). While using the advantages of UAVs, the platform realizes the coordinated interconnection of the tablet (or mobile phone), server, and UAV. It can simultaneously plan and control the UAV on the tablet. Real-time online map editing and data transmission to the server in the local area network can easily establish remote sensing field interpretation signs and complete field review operations.

2.1.2 Near real time evaluation of water and soil loss with UAV: This technology utilizes the function of real-time data acquisition by UAVs, performs digital terrain analysis on the basis of data and assists in the improvement of the original water and soil loss evaluation system. The difficulty of calculating slope and aspect, and the key factors of water and soil loss can be evaluated in the field, which has the advantages of accuracy, automation and real-time. It also proposes the UAV collaborative visual deformation monitoring technology without GNSS signals and control points, which can complete the UAV monitoring tasks under difficult conditions.

\subsection{Indoor dynamic interpretation}

1) GRSCM (Geometric and Radiometric Simultaneous Correction Model) framework for remote sensing images preprocessing: There are some geometric deformation and radiometric deformation in remote sensing images. They require manual correction processing in the later period and take a long time, resulting in a time difference between field data and image data, reducing efficiency and accuracy. The grey value $g(x, y)$ of pixel on radiometric spectrum is regarded as a function of the geometric coordinates $(x, y)$. Hence, there is a unity of opposite relationships between the geometric and radiometric information, such that, these two types of information cannot be separated ( $\mathrm{Li}$ and Xiong 2017). We have proposed a novel geometric and radiometric simultaneous correction model (GRSCM) framework inspired and developed from least squares matching (LSM). It uses a combination of random sample consistency (RANSAC), stepwise regression, and significance test. The model framework can provide accurate geometric coordinates and radiation correction information for remote sensing change detection, thereby improving the quality of dynamic monitoring.

2) Quality control method of an object-oriented land use change update supported by multi-PC and Random forest: In the process of using Deep Learning, using random forest to train a large number of samples can realize the automatic classification of massive data. This paper proposes to combine the two technologies of multi-PC terminal and random forest classifier to perform Deep Learning on a large amount of remote sensing image data. Random forest is an algorithm that gathers multiple decision trees. A decision tree is a classifier, each sample data gets $\mathrm{N}$ results in the random forest, and the category with the highest number of times is output as the final recognition result. Multiple computers extract the features of sample data and optimize it. Random forest can automatically repair topology errors and merge small patches on remote 
sensing images that need to be interpreted, and compare and classify features based on previous sample training results. Experiments show that the random forest method can obtain better classification accuracy, properly remove some redundant and related features, thereby effectively improving classification accuracy.

3) Object oriented remote sensing image classification system supported by GIS and Deep Learning: Deep learning method shows its advantage on collecting such information from remotely sensed images while requiring sufficient training sample (Xu et al. 2019) the computer can recognize a large number of remote sensing image data through Deep Learning. CNN can extract image features effectively. The fusion of the two technologies can automatically segment the remote sensing image and generate the probability algorithm of the surface features of the deep learning model. Compared with the traditional machine recognition method, the algorithm is no longer based on a single minimum pixel in the recognition process, but takes the similar features as objects to be considered and then extracts and recognizes. It greatly improves the speed, automation and reliability of remote sensing image interpretation.

4) Change detection of three-dimensional topography and vegetation from True color image: Vegetation changes in Digital Ortho Map (DOM) are detected by the CIE Lab and image segmentation algorithms without near-infrared band. This study proposes an adaptive threshold method of threedimensional change detection based on the theory of probability and statistics. The corresponding terrain change quantities can be estimated by discretized integral. It can be performed on visible light images without near-infrared wave bands. This method can sublimate the two-dimensional change detection of conventional remote sensing to the three-dimensional space, and estimate the earthwork volume of the three-dimensional changed terrain, which is helpful for the quantitative assessment of water and soil loss intensity after geological disasters.

\section{RESULTS AND ANALYSIS}

\subsection{Overview of the experimental area}

The experimental area is one of the tributaries of the Yangtze River. The main stream of this tributary has a total length of $1037 \mathrm{~km}$. Its watershed area is $87,900 \mathrm{~km}^{2}$. The topography of the drainage basin is higher in the southwest and lower in the northeast. Due to the large height difference, the natural drop is large and the cutting effect is obvious. The remote sensing images this paper used were collected by the first highresolution optical transmission mapping satellite for civilian use ZY-3 satellite. After ortho-rectification and integration, the ground resolution of standard false color synthesis reached up to $2.1 \mathrm{~m}$. The land used data of earlier years were provided by the project for comparative analysis with the newly interpreted data. This paper makes use of the remote sensing images and assists Arc GIS to carry out technical innovations and experiments to improve the accuracy of remote sensing interpretation in internal and external industries.

\subsection{Results and discussion}

In order to verify the effect of these new technologies on the interpretation of remote sensing images, they have been successfully applied in dynamic monitoring of soil and water loss in the Yangtze River Basin, China from 2015 2019. The results show that:
1) The field investigation platform based on Internet and UAV for remote sensing interpretation is easier and faster for monitors to obtain data, and also facilitates the connection between the establishment of early interpretation signs and the later recheck work. It improves the efficiency of field work.

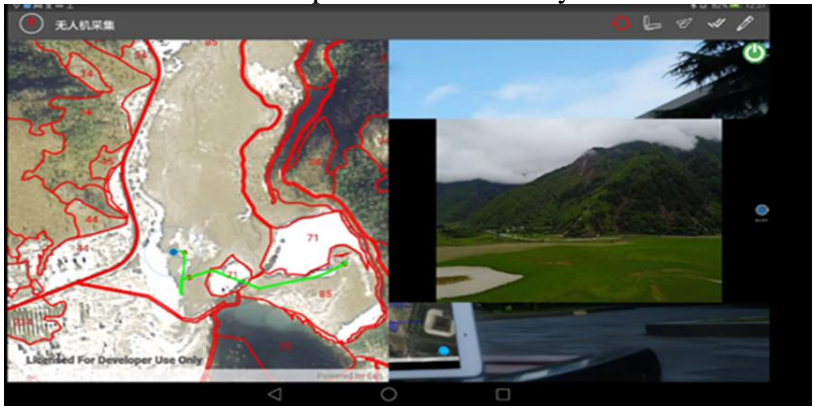

Figure 1. UAV field route planning, flight control and flat map editing interface

2) Near real-time evaluating key factors of soil and water loss based on UAV photogrammetry and digital terrain analysis are consistent with the results of traditional evaluation methods, but they are more automated and accurate. In addition, the system has low requirements on the surrounding conditions, and the monitoring task can be carried out under a variety of special conditions.

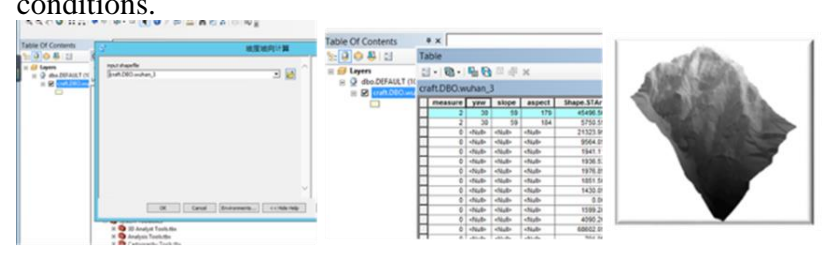

Figure 2. UAV near real time DEM generation and slope direction calculation toolbox

3) GRSCM framework for remote sensing images preprocessing makes up for the limitation of traditional artificial radiometric correction such as delay, low accuracy high error rate and so on. The experimental results demonstrate that the accuracy of the GRSCM is significantly improved compared with that of geometric correction and radiometric correction separately.

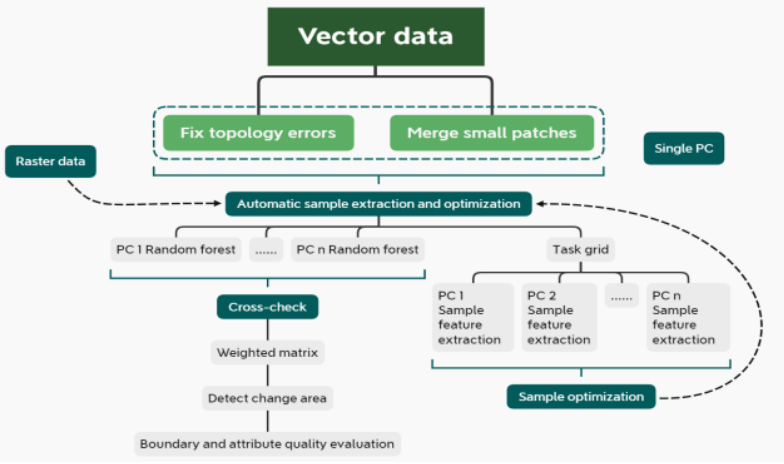

Figure 3. Quality control toolbox for object-oriented land change update supported by multiple PCs

4) Multi-PC, GIS and Deep learning support the following remote sensing interpretation system for objects, using massive data for Deep learning. Compared with the results of traditional machine interpretation, the accuracy and automation are greatly improved, and the overall accuracy can reach $94 \%$. 


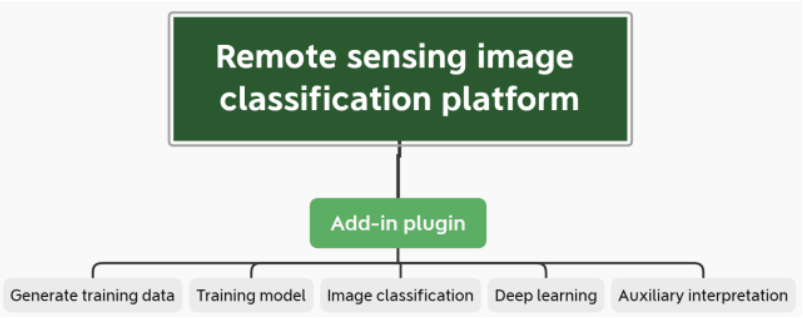

Figure 4. object oriented image interpretation toolbox based on transfer learning and deep neural network

5) Usually two-dimensional change detection is based on the premise that the elevation is unchanged, but elevation is an important information that cannot be ignored in common geological disasters. The image vegetation and threedimensional terrain quantitative change detection method integrates information such as vegetation coverage, slope and elevation in the detection area, and can achieve a more comprehensive and accurate analysis of soil and water loss after the disaster according to the specific disaster characteristics.
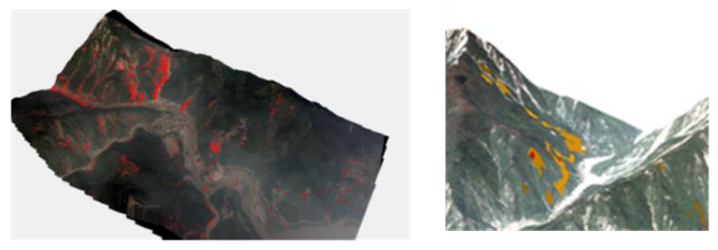

Figure 5. vegetation and three-dimensional terrain change detection in debris flow and landslide area

6 ) Figure 7 lists the accuracy of the result review in 10 experimental areas. Overall, the accuracy is maintained at about $94.3 \%$, and the accuracy of each area is not less than $90 \%$. The experimental results show that the use of the above new technologies improve the accuracy of dynamic monitoring of soil and water loss in the Yangtze River and interpretation of remote sensing images.

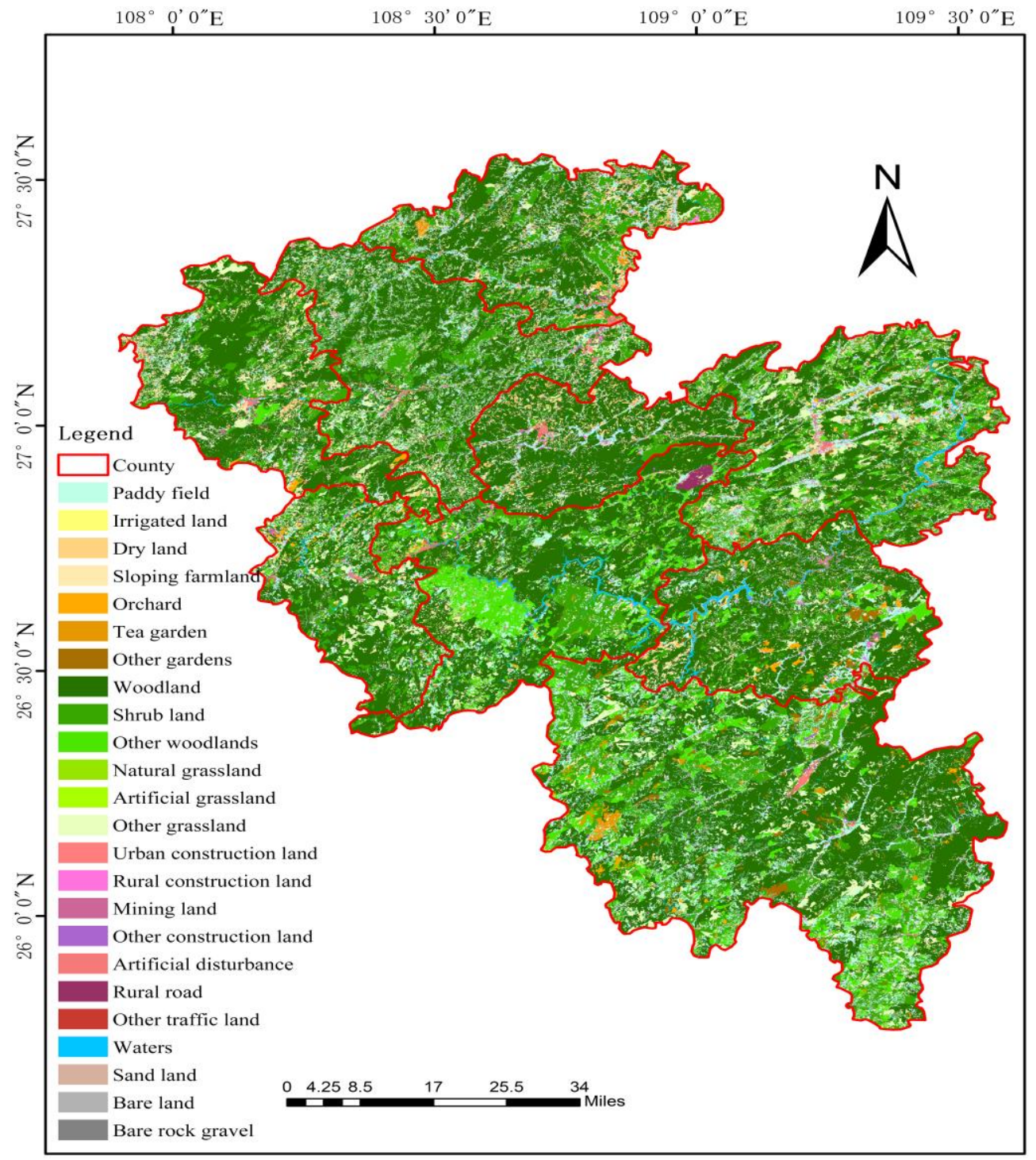

Figure 6. Thematic map of remote sensing image interpretation of one of the tributaries of the Yangtze River 


\begin{tabular}{|c|c|}
\hline $\begin{array}{c}\text { Region } \\
\text { Number }\end{array}$ & $\begin{array}{c}\text { Accuracy } \\
(\%)\end{array}$ \\
\hline 1 & 94.90 \\
\hline 2 & 94.20 \\
\hline 3 & 94.77 \\
\hline 4 & 93.70 \\
\hline 5 & 94.30 \\
\hline 6 & 93.49 \\
\hline 7 & 94.53 \\
\hline 8 & 94.55 \\
\hline 9 & 93.71 \\
\hline 10 & 94.83 \\
\hline
\end{tabular}

Tab. 7 statistical table of the accuracy of review results in some test areas

\section{CONCLUSION}

During the process, the operation is more convenient and the results are more accurate. In addition, new technologies will be able to provide technical services for the dynamic monitoring of soil erosion in other river basins.

Taking the Yangtze River Basin as an example, based on the technical problems encountered in the dynamic monitoring of soil and water loss in the Yangtze River Basin for many years, this paper puts forward the key technologies of remote sensing interpretation of the internal and external work of the dynamic monitoring of soil and water loss in the Yangtze River Basin. This paper overcomes key $3 \mathrm{~S}$ technologies for soil and water loss dynamic monitoring. These techniques are more convenient and accurate in the process of practical monitoring and interpretation. They will provide decision-making basis for government scientific management and deepen the promotion of development of "the Yangtze river economic zone and the road of sustainable development". This study will be able to produce huge ecological, economic and social benefits. The research results will provide technical experience and reference for further prevention and control soil and water loss in other rivers and will create a green China and a healthy China.

\section{ACKNOWLEDGEMENTS}

The authors thank the technical services of remotely sensed interpretation for dynamic monitoring water and soil loss in the national key controlling areas of Yangtze River Basin, China (YZJ2015-022), National Natural Science Foundation of China (NSFC) (Grant No. 41771493 and 41101407), and selfdetermined research funds of CCNU from the basic research and operation of MOE (Grant No. CCNU19TS002) for supporting this work. The authors are grateful for the comments and contributions of the anonymous reviewers and the members of the editorial team.

\section{REFERENCES}

Cutler, D. R., T. C. Edwards, K. H. Beard, A. Cutler, K. T. Hess, J. Gibson and J. J. E. Lawler (2007). "Random Forests for Classification in Ecology." [J]. Ecological Society of America, 2007, 88(11).

Ketcham, R. A., W. D. J. C. Carlson and Geosciences (2001). "Acquisition, Optimization and Interpretation of X-ray Computed Tomographic Imagery: Applications to the Geosciences." [J]. Elsevier Ltd, 2001, 27(4): 381-400.
Krizhevsky, A., I. Sutskever and G. E. Hinton (2012). "ImageNet Classification with Deep Convolutional Neural Networks." [C] NIPS. Curran Associates Inc. 2012, 25(2).

Lecun, Y., Y. Bengio and G. E. J. N. Hinton (2015). "Deep Learning." [J]. Nature Publishing Group UK, 2015, 521(7553): 436-444.

Li, C., H. J. P. E. Xiong and R. Sensing (2017). "A Geometric and Radiometric Simultaneous Correction Model (GRSCM) Framework for High-Accuracy Remotely Sensed Image Preprocessing." [J]. Photogrammetric Engineering \& Remote Sensing, 2017, 83(9):621-632.

Li, C., Y. J. Zhu, G. E. Li, Y. Q. Zhu and R. H. Li (2016). "Dynamic Monitoring of Soil and Water Losses Using Remote Sensing and GIS Techniques: A Case Study of Jialing River, Yangtze River, China." ISPRS - International Archives of the Photogrammetry, Remote Sensing and Spatial Information Sciences: XLI-B8, 947-951.

Li J. Y., Huang. X., and Gong. J. (2019). "Deep Neural Network for Remote-sensing Image Interpretation: Status and Perspectives." [J]. National Science Review, 2019, 6(06):10821086.

Mao, H., L. Rui, X. Rao and T. Zhang (2011). The Research of Remote Sensing Geological Interpretation in Long-zhang Expressway of Hunan. [C]. 19th International Conference on Geoinformatics. [v.1].2011:1-5.

Oldeman, L. R. (1992). Global Extent of Soil Degradation. BiAnnual Report 1991-1992/ISRIC, ISRIC: 19-36.

Parno, M. D., B. A. West, A. J. Song, T. S. Hodgdon and D. T. O'Connor (2019). "Remote Measurement of Sea Ice Dynamics with Regularized Optimal Transport" [J] Geophysical Research Letters. 46(10).

Waite, C. E., G. M. F. van der Heijden, R. Field, D. S. Boyd (2019). "A View from above: Unmanned Aerial Vehicles (UAVs) Provide a New Tool for Assessing Liana Infestation in Tropical Forest Canopies." [J]. Journal of Applied Ecology, 2019, 56(4).

Xu, J. X., Y. Fang (2019). A Machine Learning Dataset for Large-Scope High Resolution Remote Sensing Image Interpretation Considering Landscape Spatial Heterogeneity [C] The International Archives of the Photogrammetry, Remote Sensing and Spatial Information Sciences. 2019. XLII-2-W13. 